\title{
Erratum
}

\section{Edge Pinning and Internal Phase Transitions in a System of Domain Walls}

\author{
Theodore W. Burkhardt \\ Department of Physics, Temple University, Philadelphia, Pennsylvania, USA \\ P. Schlottmann \\ Institut für Festkörperforschung der Kernforschungsanlage, \\ Jülich, Federal Republic of Germany \\ Z. Phys. B - Condensed Matter 54, 151-158 (1984)
}

In the second paragraph of page 156, the sentence "Presumably there is a latent heat of fusion associated with the order-disorder transition at $h= \pm h_{c}(U), U<-2$ if the temperature is varied at constant $\mu "$, should be deleted. Although the staggered long-range order disappears discontinuously in crossing from the ordered to the disordered phase, there is no latent heat. Exact results [28] for the ground state of the $x x z$ Heisenberg Hamiltonian imply that the order-disorder transitions are of the Pokrovsky-Talapov type [2-9]. On the disorder side of the transition temperature, the deviation of the domain-wall density from the value $n / N_{x}=1 / 2$ grows as $\left|T-T_{c}(\mu)\right|^{1 / 2}$, and the specific heat diverges as $\left|T-T_{c}(\mu)\right|^{-1 / 2}$.

We thank Michael E. Fisher for calling this to our attention and also acknowledge helpful discussions with Daniel R. Grempel.

Theodore W. Burkhardt

Department of Physics

Temple University

College of Liberal Arts

Philadelphia, PA 19122

USA
P. Schlottmann

Institut für Festkörperforschung

Kernforschungsanlage Jülich $\mathrm{GmbH}$

Postfach 1913

D-5170 Jülich 1

Federal Republic of Germany 